\title{
APPLICATION OF THERMAL IMAGING IN OPTHALMOLOGY
}

\author{
M. Menaka ${ }^{\dagger *}$, U. Manoharan, D. Sharath, P. Bhattacharjee, B. Venkatraman \\ Health Safety and Environment Group \\ Indira Gandhi Centre for Atomic Research \\ Kalpakkam, 603102 INDIA \\ +*Presenting and Corresponding Author: menaka@igcar.gov.in
}

\begin{abstract}
This study focuses on the application of thermal imaging for Ocular Surface Temperature measurement in eye. A database of Ocular Surface Temperature of normal subjects has been generated with respect to age and sex and the results are correlated to body temperature. Analysis is carried out by recording temperature of eye in different gages. Based on this experience standard protocols have been developed and the application of thermal imaging has been further extended for evaluation of Glaucoma and dilation procedure.
\end{abstract}

KEYWORDS: Thermal Imaging, Ocular Surface Temperature, Glaucoma, Diloation

\section{INTRODUCTION}

Eyes are one of the most important and delicate part of human body and hence one need to take care of them. The most leading causes of visual impairment and blindness are cataract, glaucoma, macular degeneration and diabetic retinopathy. There are a number of eye imaging systems like confocal microscopy, corneal topography, optical coherence tomography, computerized tomography, ultrasonic biomicroscopy are available to visualize noninvasively different parts of the eye with higher precision. But most of techniques cause inconvenience to the patient. It has been recognized from ancient times that temperature is one of the best indicators of human health. Infrared Thermography (IRT) is a non-invasive and non-contact temperature measurement method, used to capture real-time temperature distribution over object surfaces. With the advances in sensor and imaging technologies, thermal imaging started finding wide applications as an adjunct imaging tool in clinical diagnostics. International literature review reveals that thermal imaging has been attempted for evaluation of dry eye [1]. However, limited work has been carried out so far in the field of ophthalmology [2]. Each eye has a distinctive surface temperature profile. Till date literature survey has revealed that no significant attempt has been made to map the Ocular Surface Temperature (OST) profile. An insight into the temperature band of normal eyes is a first step in evaluation of typical eye problems. In the present study evaluation of eye surface temperature along with body temperature of an individual using IRT is carried out. Temperature measurements are evaluated in a group of 100 voluntaries with different age groups and sex-matched controls were included in the study. Based on the experienced gained, the application of IRT has been extended to other fields like Glaucoma.

\section{MATERIAL AND EXPERIMENTAL SET UP}

\subsection{Participants}

For OST measurement of normal subjects, a total of 81 healthy volunteers (40 males and 41 females) were enrolled. Body temperature was measured using a portable thermometer applied on the forehead. This parameter was measured after the test for all volunteers. All subjects were in healthy conditions, and no one was suffering from cardiovascular diseases, glaucoma or other ocular pathologies. For screening of Glaucoma study a total of 12 subjects (patients with glaucoma) and 21 subjects (normal volunteers) were evaluated. For studying the effect of dilation on OST, a total of 50 diabetic patients have been studies.

\subsection{Experimental Setup}


Images were acquired with the subject's eyes wide open using a focal plane array based IR camera (CEDIP Make) with thermal sensitivity of $25 \mathrm{mK}$ and maximum frame rate of $176 \mathrm{~Hz}$. Camera works in the spectral range from 3.6 - $5.1 \mu \mathrm{m}$. ALTAIR software of the CEDIP Infrared Systems was used for image acquisition and analysis. Thermograms were taken using same magnification and same distance from the eye at room temperature of $28 \pm$ $1.0^{\circ} \mathrm{C}$, humidity $60 \%$, and with stable airflow during the image acquisition. A chin rest is used to minimize the head movement of subjects.

\section{RESULTS AND DISCUSSION}

\subsection{OST Evaluation of Normal Subjects}

Thermal images of each eye were acquired separately with various eye gaze namely Right straight gaze, Left straight gaze, Right down gaze, Left down gaze, Right up gaze and left up gaze. Infrared image analysis was carried out in two different ways; (i) Points was placed at seven different anatomical locations namely Center of Cornea, Nasal Limbus, Nasal Conjuctiva, Temporal Limbus, Temporal Conjuctiva, Superior Region and Inferior Region (Figure 1(a)) (ii) In another scheme, a horizontal line running through centre of cornea, connecting nasal and temporal regions (Figure 1(b)). Typical temperature profile of left and right eye is shown in figure 2 which clearly differentiates the locations in eye.
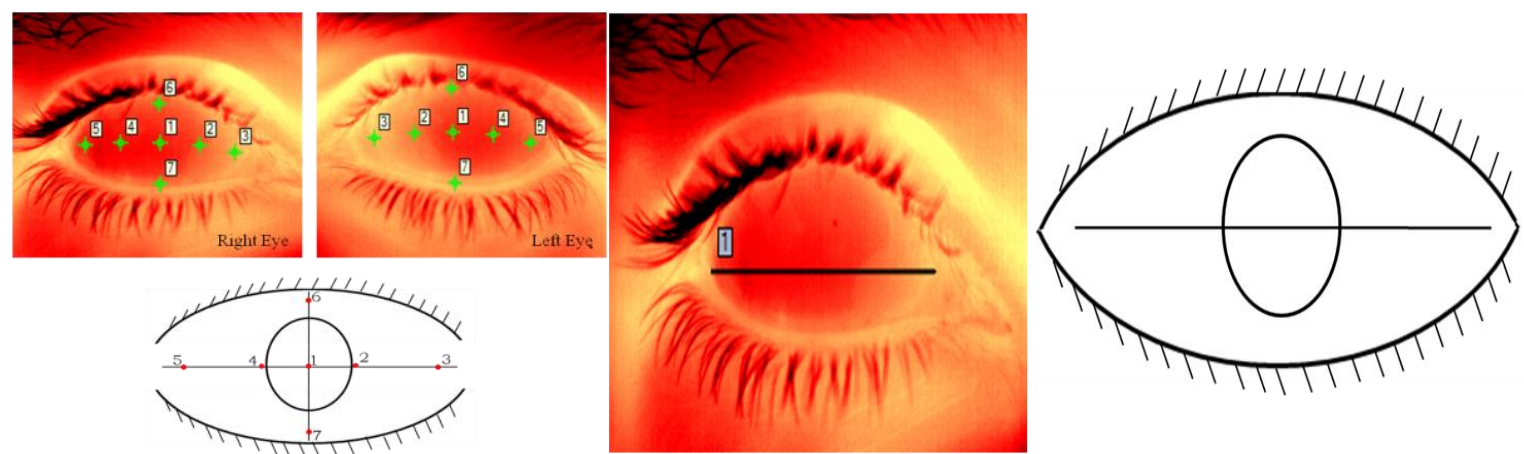

Fig. 1 Schematic diagram of eye showing two schemes used for evaluating OST using thermography

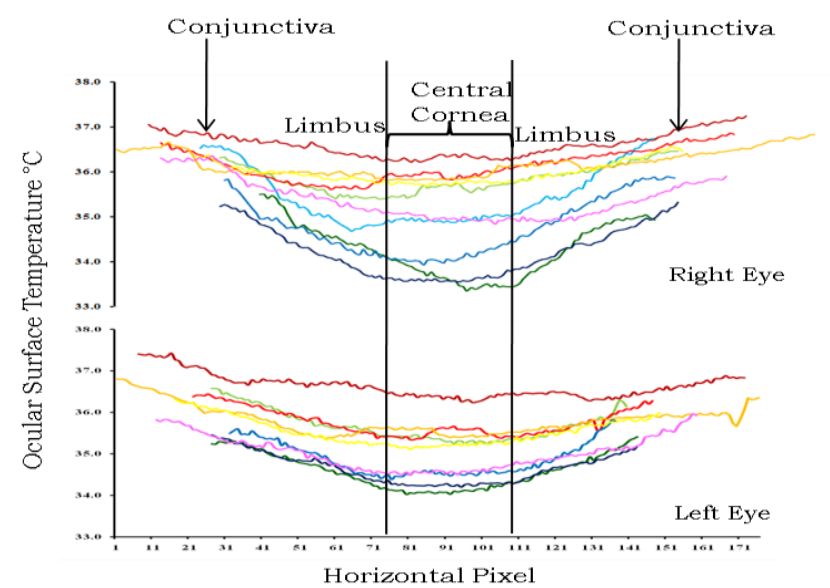

Fig. 2 Typical temperature profile of eye

\subsection{Evaluation of Glaucoma and Dilation Process}

Based on the experienced gained on normal subjects the OST measurement was extended for evaluation of Glaucoma in eye and dilation process in diabetic subjects. Figure 3 shows the thermal image of diabetic subjects before and after dilation showing clear increase in OST. In case of Glaucoma also an increase in OST was observed when compared to normal OST. 

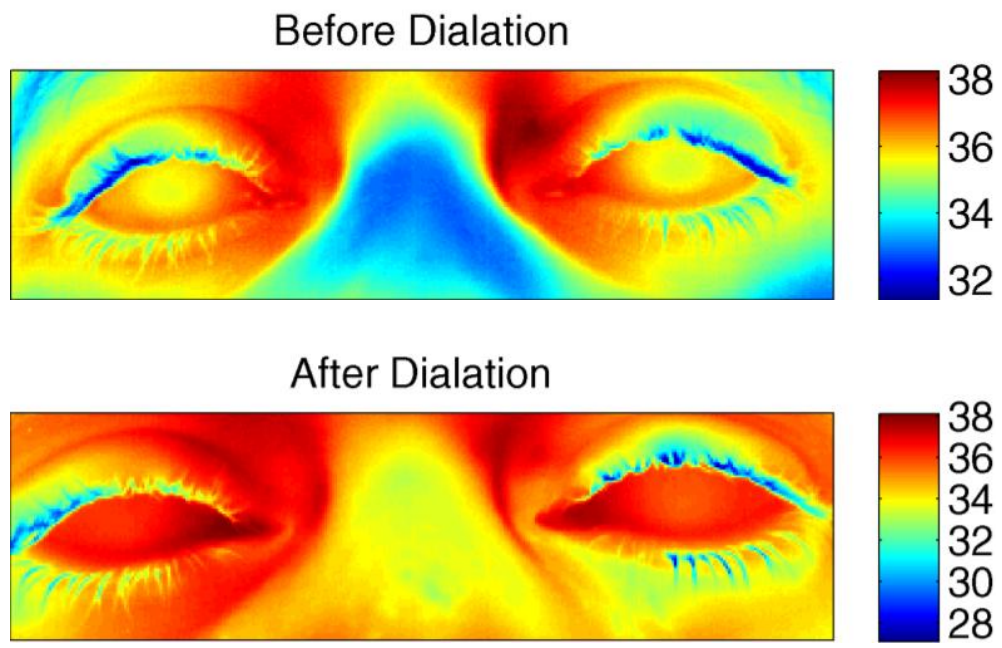

Fig. 3 Thermal image of a diabetic subject before and after dilation

\section{CONCLUSION}

Thermal Imaging was successfully applied for evaluation of Ocular Surface Temperature in human eye. OST was evaluated for normal subject and was correlated with body temperature. A lower body temperature has been reported when compared to OST. Thermal imaging also successfully applied for Glaucoma and dilation process evaluation in eye. The results indicated the potential of thermal imaging as diagnosis tool in the field of Ophthalmology.

\section{ACKNOWLEDGMENT}

We are thankful to Mr. Gnanmurthy and Mr. S. Aadithya for their assistance during the experiment. We also thank Dr. A. K. Bhaduri, Director IGCAR \& GSO for his consistence encouragement and support.

\section{REFERENCES}

[1] Morgan PB, Tullo AB, Efron N. Infrared thermography of the tear film in dry eye. Eye, 09, 1995: 615-618.

[2] Acharya R, Yun WL, Ng EY, Yu W, Suri JS. Imaging systems of human eye: a review. Journal of medical systems. 32(4), 2008:301-315. 